\title{
Projekt „Planspiel Strafprozessrecht - von der Tat zum Urteil“
}

\author{
Marc Reiß*
}

\section{A. Einleitung}

Ziel der Veranstaltung war es, für Studenten ${ }^{1}$ nach dem Grundstudium exemplarisch den Gang eines Strafverfahrens ab der Tat und bis zum Urteil als Planspiel erfahrbar zu machen. Nachdem sie wöchentlich typische Aufgaben des strafprozessualen Verfahrens aus der Sicht je eines der Prozessbeteiligten (Richter, Staatsanwalt, Verteidiger) zu bewältigen hatten, konnten die Teilnehmer in einem (simulierten) Strafprozess am Ende des Semesters eine Hauptverhandlung selbst durchführen und abschließen.

Die Vorbereitungsphase war als E-Learning-Einheit gestaltet, die mit wenigen Präsenzsitzungen und wöchentlicher Kleingruppenarbeit Lerninhalte im Wege des problemorientierten Lernens („POL“) aufgriff.

\section{B. Zur Entstehung des Projekts}

Das Projekt entstand als Gruppen-Abschlussarbeit zu einer eLearning-WorkshopReihe der zentralen eLearning-Einrichtung ${ }^{2}$ der Goethe-Universität Frankfurt. Es war im Sommersemester 2010 Gegenstand eines positiv beschiedenen Förderantrags, wurde aus später noch zu erläuternden Gründen dann allerdings nicht in der ursprünglich bewilligten Form umgesetzt. Nachfolgend wird zunächst das ursprüngliche Konzept erläutert.

\section{Zielsetzung/Ausgangslage}

Zwei Motive waren zentral bei der Planung: Erstens sollte die Veranstaltung dem häufigen Vorwurf mangelnden Praxisbezugs begegnen, dem sich die universitäre

* Der Autor ist akademischer Rat a.Z.am Fachbereich Rechtswissenschaft der Goethe-Universität Frankfurt am Main und koordiniert dort seit 2012 das Universitätsrepetitorium.

1 Um die Leserinnen und Leser dieses Artikels nicht unnötig im Lesefluss zu stören, wird auf sinnentstellende Partizipien wie „Studierende“ verzichtet (dazu etwa: Goldt, „Wenn man einen weißen Anzug anhat", S. 55), gleiches gilt für Doppelnennungen, den Gebrauch des Binnen-I oder Binnen-Unterstrichs sowie sonstige Alternativen für das generische Maskulinum - kritisch zu dessen Verwendung etwa: Posch, Mitgefangen - Mitgehangen?, S. 1 (14). Sprachpsychologische Studien belegen, dass Informationen aus geschlechtsneutral formulierten Texten zwar kognitiv ähnlich gut verarbeitet, aber subjektiv als signifikant schlechter lesbar eingestuft wurden, siehe Braun/Oelkers et al., in: PRU 2007, S. 183 (187f.). Dieser Befund deckt sich mit der überwiegend stilistisch, teils aber auch soziologisch begründeten Kritik an den Vorschlägen der feministischen Linguistik, siehe etwa: Klann-Delius, Sprache und Geschlecht, S. 186; Thurner, Der Gender-Krampf verhunzt die deutsche Sprache, Die Welt vom 2.2.2013, http://www.welt.de/debatte/kommentare/article113305194 (9.2.2014); Schneider, Thüringer Allgemeine vom 9.1.2014, http://www.thueringer-allgemeine.de/web/zgt/suche/detail/-/specific/LutherDisput-in-Erfurt-1003990437 (9.2.2014).

2 http://www.studiumdigitale.uni-frankfurt.de (9.2.2014). 
Rechtslehre immer wieder ausgesetzt sieht. ${ }^{3}$ Insbesondere der sonst so abstrakte Gegenstand der Vorlesung „Strafrecht IV - der deutsche Strafprozess“ (4. Fachsemester) sollte für Studenten des Fachbereichs nicht nur erfahrbar werden (wie etwa als Zuschauer einer Hauptverhandlung), vielmehr sollten die Teilnehmer ein Verfahren in der Rolle der juristischen Hauptakteure (Anklage, Verteidigung, Gericht) maßgeblich steuern und gestalten.

Zur gemeinsamen nachträglichen Analyse war geplant, die Verhandlung aus verschiedenen Blickwinkeln audiovisuell aufzuzeichnen. Um die Beteiligten angemessen auf die Verhandlungssituation vorzubereiten, sollten vorab strafprozessuale Inhalte behandelt werden, untergliedert in wöchentliche, individuell auf die drei Gruppen zugeschnittenen Aufgaben.

Das zweite Motivbündel fußte auf der Überlegung, die bislang beim Einsatz von eLearning äußerst zurückhaltenden und eher unerfahrenen Lehrkräfte des Fachbereichs Rechtswissenschaft zu eigenen Projekten zu motivieren und inspirieren. Die technische Umsetzung sollte dabei zunächst noch durch Externe unterstützt werden, gleichzeitig sollte hierdurch am Fachbereich aber auch ein Grundstein für eigene Kompetenzen gelegt werden, um dieses und andere Projekte künftig eigenständig durchzuführen.

\section{Zielgruppe}

Die Veranstaltung sollte sich an Studenten kurz vor dem Schwerpunktbereichsstudium richten, begleitend zur schon genannten Pflichtveranstaltung. Seminare in der Teilnehmerstärke des Projekts waren und sind im Frankfurter Schwerpunktbereich die gängige Praxis, examensrelevante Leistungsnachweise zu erlangen. 20 bis 30 Studenten sollten für die Teilnahme einen Schlüsselqualifikationsnachweis erwerben und optional eine studienbegleitende, examensrelevante Prüfungsleistung ablegen können (durch Verfassen einer projektbezogenen Seminararbeit am Ende der Veranstaltung).

\section{Inhaltliche Ausrichtung und Vorgehen im Einzelnen}

Alle Teilnehmer würden gleichmäßig auf die drei Gruppen der Prozessbeteiligten verteilt werden (Gericht, Staatsanwaltschaft, Verteidigung). Nach der Vorbesprechung und Gruppenbildung wäre eine zweite Präsenzphase dem zugrundeliegenden Sachverhalt gewidmet gewesen, so wie er an die Verfahrensbeteiligten auch in der Realität herangetragen würde (Mandantenkontakt, Übersenden polizeilicher Ermittlungsergebnisse, ggf. Anträge an das Gericht im Ermittlungsverfahren). In den On-

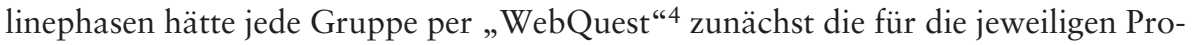
zessbeteiligten rollenspezifischen Inhalte erarbeitet und sich dann in der Gruppe auf

3 Differenziert kritisch zu diesem Vorwurf Hufen, in: ZDRW 2013, S. 5 (9).

4 Grundlegend zu dieser Methode: Wagner, S. 1 (1); überblicksartig: Gerber, Webquests für Eilige, http:// www.webquests.de/eilige.html (9.2.2014). 
eine bestimmte Vorgehensweise einigen müssen. In der Phase des Ermittlungsverfahrens etwa wäre es an der Staatsanwaltschaft gewesen, Ermittlungsmaßnahmen festzulegen und eine Anklageschrift zu formulieren, die Verteidigung hätten eine Verteidigungsstrategie ersinnen und das Gericht über Anträge entscheiden müssen. Im gespielten Prozess sollten dann alle Akteure individuell nach Gruppen gefilmt werden, um ihnen die Möglichkeit zu geben, ihre Handlungen aus Fremd- und Eigenwahrnehmung zu reflektieren. ${ }^{5}$ In der abschließenden Präsenzsitzung wäre das Urteil verkündet, ein Fazit gezogen und das Vorgehen der einzelnen Gruppen analysiert worden.

\section{Ursprünglich geplantes didaktisches Szenario}

Die Struktur des Konzepts folgt den Grundsätzen des Blended Learnings: 6 Drei ganztägige Präsenzveranstaltungen sollten durch sechs Onlinephasen verbunden sein. Als vorrangige eLearning-Methode wären „WebQuests“ zum Einsatz gekommen, zur Selbstkontrolle des Wissensstandes waren Online-Quizfragen vorgesehen. Die Lerninhalte wären online- und präsenzphasenübergreifend, die Teilnehmer sollten das angeeignete Wissen in Kleingruppendiskussionen und bei der praktischen Anwendung vertiefen.

Erhofft war, dass die Kombination aus Präsenzveranstaltungen und Onlinephasen im Vergleich zu „,herkömmlichen“ Lehrveranstaltungen organisatorische, didaktische und inhaltliche Mehrwerte aufweisen würde: Durch die Flexibilisierung von Ort und Zeit, die schnelle Verfügbarkeit von Materialien und die zentrale Erreichbarkeit sollte den Lernenden das Lernen und den Lehrenden das Lehren erleichtert werden. ${ }^{7}$ Über „WebQuests“ hätten die Studenten in einer angeleiteten Recherche an strafprozessuale Inhalte herangeführt werden können, mit den Quizfragen wären sie in der Lage gewesen, jederzeit selbstständig ihren Lern- und Leistungsstand zu überprüfen. Die Untergliederung nach Verfahrensbeteiligten und Prozessphasen sollte die Vorlesungsinhalte noch einmal aus neuer Perspektive vermitteln, da sich über den Stoff auch konkret die Handlungsspielräume der Akteure in den Präsenzphasen definiert und somit die Lerninhalte auch einen direkten, persönlichen Bezug gehabt hätten.

\section{Verändertes Konzept}

\section{Unvorhergesehene Ereignisse}

Schon in der Vorbereitungsphase zur ersten Veranstaltung kam der Vorschlag auf, die ursprünglich angedachte Methode des „WebQuests“ durch eine andere, aktivierendere Methode zu ersetzen. Relativ schnell fiel die Wahl auf das problemorientierte

5 Speziell zu dieser Funktion der Auswertungsphase bei Planspielen: Geuting, in: Blätte/Herz (Hrsg.), S. 15 (39f.).

6 Dazu ausführlich m.w.N.: Bremer, in: Bloh/Lehmann (Hrsg.), S. 175 (186f.).

7 Bremer, in: Bloh/Lehmann (Hrsg.), S. 175 (175). 
Lernen, ${ }^{8}$ hauptsächlich aufgrund folgender erwarteter Vorzüge: ${ }^{9}$ Die Teilnehmer würden wöchentlich nicht alleine, sondern in Kleingruppen arbeiten, was auch für das Rollenspiel Vorteile erhoffen ließ, die Lehrenden würden Gelegenheit haben, den Fortschritt der Gruppe direkt zu beobachten und hätten bei Bedarf unterstützende Hinweise geben können. Auch versprach das intensive und eigenständige Erarbeiten der Lerninhalte in der Gruppe nachhaltigere Lernerfolge. Am grundlegenden Ablauf würde sich nichts ändern, es müsste lediglich eine weitere Präsenzsitzung für das Erklären der Methode eingeplant werden.

\section{Verzögerungen bei der Ausarbeitung/Zusammenstellung der Inhalte}

Durch teils private, teils dienstliche Gründe, die in keinem Zusammenhang zur Veranstaltung oder der Planung standen, fielen im Laufe der Planungsphase nach und nach drei der vier ursprünglichen Veranstalter aus. Es war offensichtlich, dass das Projekt auf keinen Fall im festgelegten Zeitraum und mit dem beantragten Konzept würde durchgeführt werden können. Mit dem Drittmittelgeber wurde daher vereinbart, die Veranstaltung zweimal zu halten, mit einer „Generalprobe“ ohne Videoaufzeichnung im Sommersemester 2011 und schließlich der „Premiere“ im Wintersemester 2011/2012 mit den geplanten Dreharbeiten. So sollte ausreichend Zeit gewonnen werden, um die aufwendige Vorbereitung der Videoaufnahmen zu treffen.

Schon in der Vorbesprechung zur ersten Veranstaltung deuteten die Interessenten an, dass sie sich davor scheuten während der Verhandlung auf Video aufgenommen zu werden, und das obwohl für diesen Durchlauf noch keine Aufnahmen geplant waren. Als Gründe wurden einerseits die ohnehin schon bestehenden Unsicherheiten im Strafprozessrecht genannt, zum anderen ein antizipiertes Unbehagen, da man beim Spielen seiner zugewiesenen Rolle vor dem Dozenten ohnehin schon sehr nervös sei und das Filmen dies wohl noch steigern würde. Diese Äußerungen wiederholten sich in der Vorbesprechung des nächsten Semesters leider in noch intensiverer Form - die potentiellen Teilnehmer äußerten trotz großen Interesses an der Veranstaltung unisono und ausdrücklich, dass sie nur teilnehmen wollten, wenn keine Aufzeichnung stattfände. De facto stand oder fiel damit die rechtzeitige Durchführung im Rahmen des Förderungszeitraums mit dem Verzicht auf das Filmen. In Absprache mit dem Drittmittelgeber wurde schließlich die Fördersumme reduziert und die Videoaufzeichnung schweren Herzens aus dem Konzept gestrichen.

\section{Tatsächlicher Ablauf}

Um die Darstellung klarer zu gestalten, sind nachfolgend erster und zweiter Durchlauf zusammengefasst, Abweichungen zwischen beiden Veranstaltungen sind kenntlich gemacht.

8 Zum Begriff allgemein: Zumbach, PBL, S. 19.

$9 \mathrm{Zu}$ den positiven Effekten des PBL etwa: Kiiver, in: Brockmann/Dietrich et al. (Hrsg.), S. 160 (160 und 165 f.); skeptischer und differenzierter bezüglich der einzelnen zugeschriebenen Vorteile m.w.N. hingegen: Harbach, Problemorientierung und Vernetzung in kontextbasierten Lernaufgaben, S. $21 \mathrm{ff}$. 


\section{Erste Präsenzsitzungen: Organisatorisches und Erklären der Lernmethoden}

Gegenstand der ersten Präsenzsitzung waren alle organisatorischen Eckdaten (Nutzung der Lernplattform, Voraussetzungen des Scheinerwerbs, zeitlicher Ablauf, Terminfindung für das Rollenspiel, Benotung), danach haben die Kursmitglieder sich nach Interessenschwerpunkt auf die Gruppen verteilt. Außerdem wurde ausführlich die Methode des „POL “ mit ihren einzelnen Phasen und Zielen ${ }^{10}$ erklärt. Hier war angemessen zu berücksichtigen, dass Studenten der Rechtswissenschaft in aller Regel mit dieser Lernmethode nicht vertraut und ihr gegenüber möglicherweise auch skeptisch eingestellt sind. Sehr deutlich waren deswegen die methodischen Besonderheiten zu erklären: dass etwa die Arbeit in Kleingruppen besonderen Dynamiken ausgesetzt sein kann, dass die Methode dazu dient, Lernziele selbstständig zu ermitteln und auf sie hinzuarbeiten, vor allem aber, dass der Dozent nur sehr passiv und höchstens als Moderator durch Fragen und zurückhaltende Anregungen in diesen Lernprozess eingreifen würde. ${ }^{11}$ Interessant war die mehrfach (und später auch im Evaluationsbogen) geäußerte Beobachtung der Teilnehmer, die große Freiheit bei der selbstbestimmten Auswahl der Lerninhalte auch als große Verunsicherung zu empfinden.

\section{Wöchentliche Aufgabe mit rollenzentriertem Aufgabendesign}

Die wöchentlich vergebenen Aufgaben bestanden überwiegend aus authentischen (anonymisierten) Aktenstücken des gleichen Falls, die je nach Gruppe unterschiedlich zusammengesetzt waren: Während die Richtergruppe etwa die Vorführung eines Verdächtigen beim Haftrichter vorzubereiten hatte, wurde der gleiche Sachverhalt der Verteidigung als nächtlicher Mandantenanruf aus der Untersuchungshaft vermittelt, der Staatsanwaltschaft in Form einer von der Polizei übersendeten Ermittlungsakte. Die ersten wöchentlichen Gruppensitzungen fanden mit dem Dozenten statt, der aber nur beobachtend teilnahm und lediglich vereinzelt durch offene Fragen eigene Überlegungen der Gruppe anstieß - jedenfalls, soweit es nicht um die Ausführung der Lernmethode ging.

Ihre Vorschläge zum Vorgehen in der jeweiligen Aufgabenstellung haben die Gruppen wöchentlich in der Lernplattform hochgeladen. Hierauf gab es direktes Feedback des Veranstalters mit Überlegungen dazu, wie prozesstaktisch sinnvoll das Vorgehen war, ob materiell-rechtliche Fehler enthalten waren, oder ob eventuell noch Aspekte des Falls übersehen wurden. Mit fortschreitendem Verlauf des Kurses waren die Aufgaben mehr und mehr auf die Vorbereitung zur Hauptverhandlung ausgelegt.

\section{Präsenzsitzungen zur Klärung und für Rückmeldung}

Nach den ersten vier Aufgaben tauschten die Teilnehmer sich mit dem Veranstalter in einer gemeinsamen Präsenzsitzung noch einmal intensiv zu ihren Erfahrungen und Fragen hinsichtlich der Lernmethode aus, außerdem kamen vereinzelte inhaltliche Fragen zur Sprache, die nach der Eigenrecherche noch unklar waren. Beim zweiten

$10 \mathrm{Zu}$ den Phasen etwa: Marks/Thömen, in: Berendt/Voss et al. (Hrsg) S. 1 (1 ff.).

11 Becker/Friske et al., in: WiSt 2010, S. 366 (S. 367 f.). 
Durchlauf wurden hier noch stärker die Besonderheiten der Methode hervorgehoben.

\section{4. „Interviewsession“ mit Staatsanwalt}

Für den zweiten Durchlauf der Veranstaltung gewährte ein Staatsanwalt als Gastdozent Einblicke in seine Tätigkeit, die Teilnehmer konnten ausführlich Fragen stellen, insbesondere auch hinsichtlich des anstehenden gemeinsamen Besuchs einer strafrechtlichen Gerichtsverhandlung.

\section{Gemeinsamer Besuch einer Gerichtsverhandlung mit anschließender Besprechung}

Für den Gerichtsbesuch wurden mehrere Daten ausgewählt, an denen Verhandlungen zu erwarten waren, die in einer abgeschlossenen Sitzung einen sinnvollen Einblick in die strafgerichtliche Tätigkeit bieten. Nicht zuletzt weil der Verlauf einer Verhandlung schnell Überraschungen in tatsächlicher Hinsicht bereiten kann (etwa das Fernbleiben des Angeklagten oder eines zentralen Zeugen, sonstige Vertagungen, überraschende Geständnisse etc.), geschah dies in enger Absprache mit der Staatsanwaltschaft. Wie alle Teilnehmer ausdrücklich bestätigten, gaben die besuchten Verhandlungen bei beiden Veranstaltungen ausreichend Anlass zu einem intensiven Austausch über typische strafprozessuale Vorgänge.

\section{Gerichtsverhandlung}

Freundlicherweise stellt der Präsident des Landgerichts Frankfurt für beide Veranstaltungen einen Gerichtssaal zur Verfügung, auch die passenden Roben konnten über einen Robenverleih relativ kostengünstig beschafft werden. Schon rechtzeitig vor dem ausgewählten Termin hatten alle Teilnehmer eine Akte in jeweils angepasster Fassung erhalten. Den Angeklagten spielte ein wissenschaftlicher Mitarbeiter, die Rolle der Zeugen übernahmen studentische Hilfskräfte, welche die Dozenten vorher entsprechend auf ihre Aussage vorbereitet hatten. Der Dozent führte das Verhandlungsprotokoll. Schon vorab war vereinbart worden, dass ein „Stuhlurteil“12 nicht erforderlich wäre, wohl aber Plädoyers von Staatsanwaltschaft und Verteidigung.

\section{Urteil und Auswertung}

In der letzten Präsenzsitzung schilderten die Teilnehmer zunächst die Eindrücke, die sie aus der Hauptverhandlung mitgenommen hatten, sowohl hinsichtlich ihrer eigenen Rollen, als auch die der anderen Gruppen. Staatsanwaltschaft und Verteidigung gaben außerdem eine Einschätzung zu dem ihnen noch unbekannten Urteil ab, das dann verlesen und in der Gruppe diskutiert wurde. Erst danach kommentierten die Dozenten das Urteil, dabei standen weniger materiell-rechtliche Inhalte im Vordergrund (die Verhandlung verlief überwiegend rechtsmittelfest), sondern vorrangig Abweichungen zur üblichen Praxis vor Gericht. Hier hatten die Teilnehmer auch Gelegenheit, ihr Rollenverständnis zu analysieren und zu hinterfragen.

12 Mit dem Begriff wird ein Urteil bezeichnet, das im Lauf der Verhandlung ergeht. 
Wer einen Seminarschein erwerben wollte, hatte danach drei Themenvorschläge einzureichen, die in Zusammenhang mit strafprozessualen Fragen stehen, aus denen der Veranstalter einen geeigneten auswählte oder jedenfalls zum Anlass nahm, eine thematisch verwandte Themenaufgabe zu stellen, die in der üblichen Bearbeitungszeit zu bewältigen war.

\section{Finanzierung}

Aufgrund der oben schon geschilderten Änderungen des Konzepts verringerte sich der finanzielle Aufwand des Projekts erheblich, die tatsächlich in Anspruch genommene Fördersumme reduzierte sich um etwa die Hälfte. Der Großteil der restlichen veranschlagten Summe entfiel auf Personalkosten (Mitarbeiterstunden zur Betreuung, Planung, Koordination), nur ein geringer Teil hingegen auf sonstige Sachmittel, namentlich die Roben. Wichtige Argumente für die Erteilung der Förderung waren, dass der Fachbereich sich bereit erklärt hatte, etwa die Hälfte der Kosten mitzutragen, dass das Projekt eine Vorreiterrolle spielen sollte, sowie der geplante Einsatz der gleichen eLearning-Methoden auch für andere Veranstaltungen des Fachbereichs.

\section{E. Auswertung}

\section{Evaluation}

Beide Veranstaltungen wurden per Fragebogen mit rund 35 Items und zwei Freitextfeldern („Besonders gut/besonders schlecht war...“) evaluiert. Anzukreuzen war nach Schulnoten von 1 bis 6 (plus Feld „keine Angabe“), um sicherzustellen, dass auch bei Noten im Mittelfeld eindeutig positive oder negative Trends zu erkennen waren. $\mathrm{Zu}$ den zentralen Fragen wurden mindestens zwei inhaltliche ähnliche oder sich überschneidende Items formuliert.

Abgefragt wurden:

- zur Veranstaltung allgemein: Gruppengröße, Engagement der Gruppenmitglieder, klare Formulierung der Lernziele, Unterstützung durch den Veranstalter, erkennbare Stoffgliederung, Interesse an den behandelten Themen

- zur Methode des POL: verständliche Erklärung, Kommunikation und Arbeit in der Gruppe, Geeignetheit der Methode und der ausgewählten Probleme, Einschätzung der Lerneffektivität

- zu den Kursinhalten: Anforderungsniveau, Geeignetheit zur Vorbereitung auf das Rollenspiel/zum Fördern des Verständnisses strafprozessualer Inhalte, Bewertung des Veranstalterfeedbacks zur Nachbereitung

- zum Rollenspiel: Qualität der Vorbereitung, Intensivität der Einblicke in strafprozessuale Abläufe und Aufgaben der Verfahrensbeteiligten, Notwendigkeit einer Videoaufzeichnung/intensiveren Nachbesprechung

- zur Veranstaltung im Ganzen: förderlich für Interesse am Stoff, beobachteter Lernerfolg, Vertiefung des inhaltlichen Verständnisses, „Lohnen“ der Teilnahme, Weiterempfehlung. 
Erfreulich ist zunächst festzuhalten, dass alle Befragten uneingeschränkt (Note $1=$ „trifft voll zu“) die Veranstaltung weiterempfehlen würden und dies selbst dann, wenn sie die Inhalte weder neu oder interessant fanden (= Note 4), noch das Gefühl hatten, mit der POL-Methode effektiver zu lernen als sonst (= Note 4 oder 5 ). Ebenfalls interessant ist die Korrelation zwischen der vermeintlichen Lerneffektivität der Veranstaltung und der eigenen Einschätzung der Lernerfolge. Denn obwohl die Lerneffektivität des POL von keinem der Teilnehmer mit mehr als Note 3, überwiegend sogar mit Note 4 oder 5 bewertet wurde, und rund 70 Prozent sich mehr Feedback seitens des Veranstalters auf die Nachbearbeitung der Kursaufgaben wünschten, fühlten sich die gleichen Befragten dennoch gut bis sehr gut vorbereitet auf das Rollenspiel und bewerteten ihren Lernerfolg und ihr Verständnis des Stoffes ebenso. Eine naheliegende Interpretation und Erklärung dieses Ergebnisses könnte fachkulturell bedingt sein und in dem teils sogar offen geäußerten Unbehagen angesichts neuer Lern- und Lehrmethoden liegen. Aus den Angaben im Freitextfeld und der Evaluation ergibt sich, dass die Methode verständlich und umfangreich erklärt (Note 1 und 2) und damit wohl auch verstanden wurde, inklusive der oben schon benannten Besonderheiten (s.o. C III. 1.). Insofern spricht vieles dafür, dass es den Teilnehmern schlichtweg an einem ausreichenden Vertrauen in die neuen Methoden fehlte und ihr Lernverständnis wohl stark geprägt war von einer Vorstellung des Veranstalters als alleinige Autorität hinsichtlich der Lerninhalte. Das führt zu der Frage, ob die juristische Fachdidaktik nicht mehr Sorge dafür tragen muss, den Sinn und Nutzen neuer Lernmethoden nicht nur hinreichend transparent darzustellen, sondern auch besonders intensiv hervorzuheben und jedenfalls intensiver als in anderen Fächern.

\section{Erkenntnisse}

Es ist nicht von der Hand zu weisen, dass die Veranstaltung jedenfalls mit Blick auf das ursprüngliche Konzept mit einigen Enttäuschungen verbunden war, allen voran die nicht geglückte Umsetzung des sehr viel aufwendigeren ersten Konzepts - zumal dies an äußeren Umständen scheiterte, die in keinem unmittelbaren Zusammenhang mit (fach-)didaktischen Fragen standen. Auch blieb der erhoffte umfassende Initialprozess am Fachbereich aus, obwohl Lehrende vereinzelt eLearning-gestützte Methoden in ihre Veranstaltungen aufnahmen. Immerhin konnten für das zu dieser Zeit noch in der Planung begriffene Universitätsrepetitorium erste grundlegende Erfahrungen für den Einsatz von eLearning-Plattformen gesammelt werden.

Ausgesprochen positiv ins Gewicht fällt hingegen die nahezu einhellig begeisterte Rückmeldung nach Abschluss der Veranstaltung. Eine der häufigsten Äußerung der Teilnehmer nicht nur in den Evaluationsbögen war, durch das Rollenspiel einen interessanten, erkenntnisreichen und für das sonstige Studium einmaligen Einblick in die berufliche Tätigkeit von Gericht, Staatsanwaltschaft und Verteidigung gewonnen zu haben. Charakteristisch dafür war nicht zuletzt der große Eifer, mit dem sie (trotz 
aller im Vorfeld ersichtlicher Nervosität) ihre Rollen in der Hauptverhandlung ausfüllten.

Als zukünftige Perspektive wäre auch an eine noch intensivere Einbindung von Praktikern zu denken, etwa als individuelle Betreuer der einzelnen Gruppen, auch mit häufigeren Rollenspielen im Laufe eines Semesters. Aus fachdidaktischer Sicht haben sich deutlich die Vorteile von POL und „,blended learning“ gezeigt: Zwar war die Ausarbeitung der Aufgaben mitunter mit etwas größerem Aufwand verbunden, dies wurde jedoch durch geringeren zeitlichen Aufwand im Laufe des Semesters wieder ausgeglichen. Mögen sie dies auch nicht bewusst wahrgenommen haben, sprechen die Lösungsvorschläge der Teilnehmer und ihr Auftreten in der Hauptverhandlung doch sehr dafür, dass die angewandte Lernmethode den an sie gestellten Erwartungen gerecht wurde. Aus Sicht des Dozenten schienen die Teilnehmer deutlich interessierter daran, praktikable und sinnvolle Lösungen für die gestellten Aufgaben zu finden, als bei einer "gewöhnlichen“ Fallbearbeitung, mit einem schärferen Blick für die sozialen und tatsächlichen Folgen ihres Handelns - möglicherweise auch angeregt durch die Fallauswahl: Eine der Aufgaben thematisierte etwa einen typischen Fall häuslicher Gewalt.

Auch zeigte sich, welche zentrale Rolle dem Instruktionsdesign zukommt - bei aller Begeisterung für neue didaktische Methoden sollten Lehrende die in der rechtswissenschaftlichen Fachkultur angelegte Strukturträgheit nicht unterschätzen und ihr intensiv entgegenarbeiten.

\section{F. Zusammenfassung}

Der Werkstattbericht gibt einen Einblick in das Konzept und die Durchführung eines strafprozessualen Planspiels und erläutert gewonnene Erkenntnisse - namentlich zur Planung und Anwendung von „blended learning“, zum problemorientierten Lernen, zu auf die Rechtspraxis ausgerichteten Lerneinheiten und nicht zuletzt zur Eigenwahrnehmung der Studenten im Rahmen solcher neuer Lernkonzepte.

\section{Literaturverzeichnis}

Becker, Fred/Friske, Vanessa/Meurer, Cornelia/Ostrowski, Yves/Piezonka, Sascha/Werning, Ellena, Einsatz des Problemorientierten Lernens in der betriebswirtschaftlichen Hochschullehre, in: WiSt (Wirtschaftswissenschaftliches Studium) 2010, S. 366-371.

Braun, Friederike/Oelkers, Susanne/Rogalski,Karin/Bosak, Janine/Sczesny, Sabine, „Aus Gründen der Verständlichkeit...": Der Einfluss generisch maskuliner und alternativer Personenbezeichnungen auf die kognitive Verarbeitung von Texten, in: PRU (Psychologische Rundschau) 2007, S. 183-189.

Bremer, Claudia, Handlungsorientiertes Lernen mit Neuen Medien, in: Bloh/Lehmann (Hrsg.), OnlinePädagogik, Bd. 2, Hohengehren 2005, S. 175-197.

Geuting, Manfred, Soziale Simulation und Planspiel in pädagogischer Perspektive, in: Blätte/Herz (Hrsg.), Simulation und Planspiel in den Sozialwissenschaften. Eine Bestandsaufnahme der internationalen Diskussion, Münster 2000, S. 15-62.

Goldt, Max, Wenn man einen weißen Anzug anhat: Ein Tagebuch-Buch, Hamburg 2002.

Harbach, Andrea, Problemorientierung und Vernetzung in kontextbasierten Lernaufgaben, Berlin 2013. 
Hufen, Friedhelm, Perspektiven des rechtswissenschaftlichen Studiums, in: ZDRW 2013, S. 5-20.

Klann-Delius, Gisela, Sprache und Geschlecht, Stuttgart 2005.

Kiiver, Philipp, Problembasiertes Jurastudium in Maastricht: Praxis seit 30 Jahren, in: Brockmann/Dietrich/ Pilniok (Hrsg.), Methoden des Lernens in der Rechtswissenschaft, Baden Baden 2012, S. 160-167.

Marks, Frank/Thömen, Doris, Die Rekonstruktion der Wirklichkeit, in: Behrend/Voss/Wildt (Hrsg.): Neues Handbuch Hochschullehre. Lehren und Lernen effizient gestalten, Loseblattsammlung C 1.1, Stuttgart 2002

Posch, Claudia, Mitgefangen - Mitgehangen? Generisches Maskulinum und Normen geschlechtergerechten Sprachgebrauchs, in: Antenhofer, Christina/Schnegg, Cordula/Oberprantacher, Andreas (Hrsg.), Ringvorlesung: Kommunikation - Kunst - Politik. Perspektiven Geisteswissenschaftlicher Forschung, http://www.academia.edu/2549523 (9.2.2014).

Wagner Wolf-Rüdiger, Workshop: WebQuest - ein didaktisches Modell zur Veränderung der Lernkultur, http://nline.nibis.de/elis/forum/upload/public/moderator/wagner_webquestkonzept.pdf (9.2.2014).

Zumbach, Jörg, Problembasiertes Lernen, Münster 2003. 